\title{
CHARACTERIZATION OF LETTERFORM COMPLEXITY
}

\author{
Irma Puškarević ${ }^{1}$ (D), Uroš Nedeljković ${ }^{1}$ (D), Nace Pušnik ${ }^{2}$ (D) \\ ${ }^{1}$ University of Novi Sad, Faculty of Technical Sciences, \\ Department of Graphic Engineering and Design, Novi Sad, Serbia \\ ${ }^{2}$ University of Ljubljana, Department of Textiles, Graphic Arts and Design, Ljubljana, Slovenia
}

\begin{abstract}
The properties of typefaces are a prominent element in graphic communication. Over time, distinctive features of letterforms have evolved, influenced by the technological advancements. Today, letterforms are part of typeface units which serve as means of communication and are available in various styles. Research studies regarding the typeface legibility and the effects of semantic properties of typefaces have given a considerable insight into the effects of the distinctive features of typefaces. However, the construction of typographic stimuli design in previous research was unsystematic leading to inconsistent results and understanding of the typeface distinctive features effects. Therefore, the aim of this paper is to propose a systematic method for manipulation of the typographic stimuli in research studies. The design of the typographic test samples involved detection of the universal letterform structure and manipulation of the formal attributes of typefaces. The method of perimetric complexity was used to measure the complexity of the letterforms' formal attributes, as well as the complexity of the complete letterform. Due to diverse letterform properties, two metric systems for measuring perimetric complexity (Pelli's algorithm and morphological components function) were chosen. The analysis of the obtained results confirmed that particular formal attributes, singular or combined, are more complex than others, that is a gradient of letterform complexity was revealed. Also, the results of the letterform characterization using the second method provide slightly more conclusive results making it possible to infer which groups of formal attributes contribute to the increase of letterforms' complexity.
\end{abstract}

Key words: typography, typeface properties, letterform, complexity of form, perimetric complexity

\section{INTRODUCTION}

The visual communication is an everyday human occurrence that is operating on both the conscious and subconscious level. The graphic communication, as a subordinate of visual communication, employs much of the verbal content, hence the properties of typefaces become a prominent element in this realm of communication. The technological advancements have left a mark on the formal structure of letterforms. For instance, at the beginning of mechanization of manuscripts, type forms have been merely a copy of scribes' calligraphic writings (Nedeljković et al, 2012; Highsmith, 2012). Gradually, the letterforms have evolved, going through several stages of reform over the centuries. After the industrial revolution, the major transformation in the design of type forms took place. Classical letterforms reached "astonishing height, width, and depth" (Lupton, 2004). Naturally, the effects of the new kinds of typography begun to interest academic circles. From a point of view of the typographic discipline, letterforms can be described by a limited set of elements. Dixon refers to these characteristics as formal attributes which she defines as "the basic individual units of description that refer to a typefaces' design and construction". In her descriptive typeface classification, these formal attributes are identified as construction, shape, proportion, modeling, weight, terminals, key characteristics, and decoration. Once these key characteristics of typeface design are defined, the greater is the opportunity to investigate effects of design properties.

Scholars in the field of human vision, cognitive and experimental psychology have been inquisitive about the letter identification process which is in a correlation with the design of letterforms (Rayner, 2009; Pelli et al, 2006). Research studies on the typeface legibility have vastly contributed to the pool of knowledge on letter identification and helped understand which distinctive features of letters aid reader in discerning visually similar letterforms. A study by Fiset et al. (Fiset et al, 2008), for example, confirms the crucial importance of terminations for letter identification. Another study by Beier and Larson (2013) uses stylization of the letter skeleton to determine and confirm that particular style characteristics are more legible than others. Nedeljković et al. (2017) set the grounds for their research on the two theoretical approaches - character differentiation and familiarity. Their findings confirm that familiarity with a typeface influences reading speed, but also that the ease with which we discern one character from another is affected by the congruency of a letterform with the universal letter skeleton. These and similar research studies on typeface legibility center around letterforms designed for longer passages of text where 
variations of the letters' formal attributes are marginal. On the other hand, short passages of text (e.g. slogans, headings) are very often set in a display typeface where the form of a letter is embellished for the sake of the additional meaning production. A study by Beier et al. (2017) investigated the effects of the distinctive features of embellished letterforms and they found that embellished typefaces need to be designed in a way as to maintain the form of the common skeleton to sustain legibility. Namely, the findings of their study indicate that all the tested embellished letterforms hinder legibility. However, the particular letter features make a typeface less or more legible, hence a ranking of the distinctive features according to their legibility might be acquired.

Apart from the legibility concerns in a typeface design, additional interest of this process lies in the meaning production. Numerous studies investigated the communicative power of typefaces inferring that distinctive letter features can convey a mood, communicate attitude or attribute persona (Brumberger, 2003; Rowe, 1982; Childers et al, 2002). If one analyses the distinctive features of typefaces, one can set apart two sets of characteristics in the process of designing (Henderson et al, 2004). The first set of characteristics represent holistic descriptions that rely on perception and fall to a subjective evaluation (e.g. symmetry). The second set of characteristics are a graphic description of letterforms which can be evaluated objectively (e.g. short/tall). Along with Dixon's identifiers of formal attributes, these typeface characteristics contribute to both legibility and meaning production aspects. However, a method for a systematic manipulation of these characteristics has not been addressed. The lack of such method has resulted in the inconsistent treatment of the typographic stimuli design in the previous research and it was difficult to obtain consistent results and understanding about the effects of distinctive features. Even though the recent studies on the subject provide useful guidance (Beier et al, 2013; Nedeljković et al, 2017; Beier et al, 2017), there is still a need for a systematic method for manipulating typographic stimuli in research studies. Therefore, the aim of this paper is to propose a method for (i) resolving the issue of the inconsistent stimulus control in the research studies and (ii) formulating a list of distinctive letter features ranging between less complex to more complex forms.

\section{METHODOLOGY}

The design of the typographic test samples in this study involved detection of the universal letterform structure and manipulation of the formal attributes of typefaces.

For the purposes of detecting the universal structure, the model of the common letter skeleton was used (Frutiger, 1998). The core principle of this model is a neutral shape of a letter which can be isolated when stylistically different typefaces blend together. More precisely, when eight most commonly found typefaces (Garamond, Baskerville, Bodoni, Excelsior, Times, Palatino, Optima, Helvetica) are superimposed, the common skeleton is revealed. Software application Adobe Illustrator was selected as a tool to design test samples since it uses vector graphics to create shapes. Uppercase letter $\mathrm{H}$ was selected as a representative letterform. Once the universal structure was detected by superimposing the letter $\mathrm{H}$ of eight typefaces in Adobe Illustrator (Figure 1), an image (perceptual) metric was selected to quantitatively evaluate created universal structure as described by Nedeljković (Nedeljković, 2016).

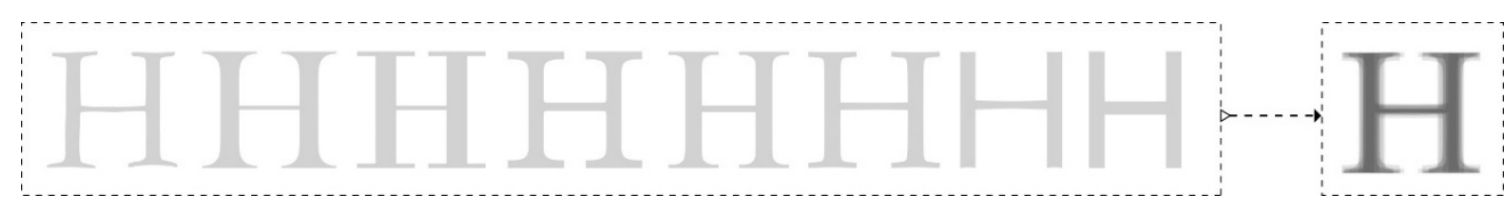

Figure 1: Construction of the universal letterform structure in Adobe Illustrator

The structural similarity index (SSIM) was selected in the ImageJ program for a quantitative sample assessment using the option of the maximum intensity. The maximum intensity of the test samples was reached by superimposing the letter $\mathrm{H}$ of eight typefaces by overlapping each pixel of one letter sample with each pixel of another letter sample on an identical $(x, y)$ position. The result of this process was an image of the letter $\mathrm{H}$ which was labelled as a referential image for the purposes of the similarity structure analysis with the previously detected universal structure. This referential structure was juxtaposed with each letter sample individually and then with the universal structure. Table 1 shows the results of the SSIM measurements where the value 1 means picture overlap 100\%. As the structure similarity index shifts to the value 0 , the difference between the two expands. The analysis of the results shows that the universal 
structure is similar with the referential structure. Thus, the universal structure is taken as a base unit for the design of the typography test samples.

Table 1: The results of the SSIM index evaluation

\begin{tabular}{|c|c|c|c|c|}
\hline Sample & Luminance & Contrast & Structure & MS-SSIM \\
\hline 1 & 0.99639 & 0.76683 & 0.83197 & 0.63567 \\
\hline 2 & 0.99040 & 0.69327 & 0.81183 & 0.55741 \\
\hline 3 & 0.98741 & 0.67588 & 0.81829 & 0.54611 \\
\hline 4 & 0.99216 & 0.67697 & 0.80208 & 0.53873 \\
\hline 5 & 0.99291 & 0.76016 & 0.85176 & 0.64288 \\
\hline 6 & 0.99409 & 0.72212 & 0.81463 & 0.58478 \\
\hline 7 & 0.99522 & 0.84861 & 0.87425 & 0.73835 \\
\hline 8 & 0.99374 & 0.79764 & 0.83417 & 0.66120 \\
\hline Univ.struc. & 0.99961 & 0.95720 & 0.94122 & 0.90059 \\
\hline
\end{tabular}

The design of the typographic samples began by designing the formal attributes. The qualitative analysis of the formal attributes that Dixon introduces in her descriptive typeface classification (Dixon, 2008) revealed that particular attributes are less complex while others are more complex. Based on this analysis it is possible to distinguish particular formal attributes (parallel strokes, rounded terminals, skewed or dashed strokes, added shadow) according to their level of complexity. Therefore, two groups of test samples were defined. The former comprises of the constituent parts of the letterform with different levels of the form's complexity (Figure 2) and the latter of the representative letter $\mathrm{H}$ constructed using the attributes from the first group (Figure 3). For the design of the test samples in both groups, the universal letterform structure was used as a reference for the starting point.

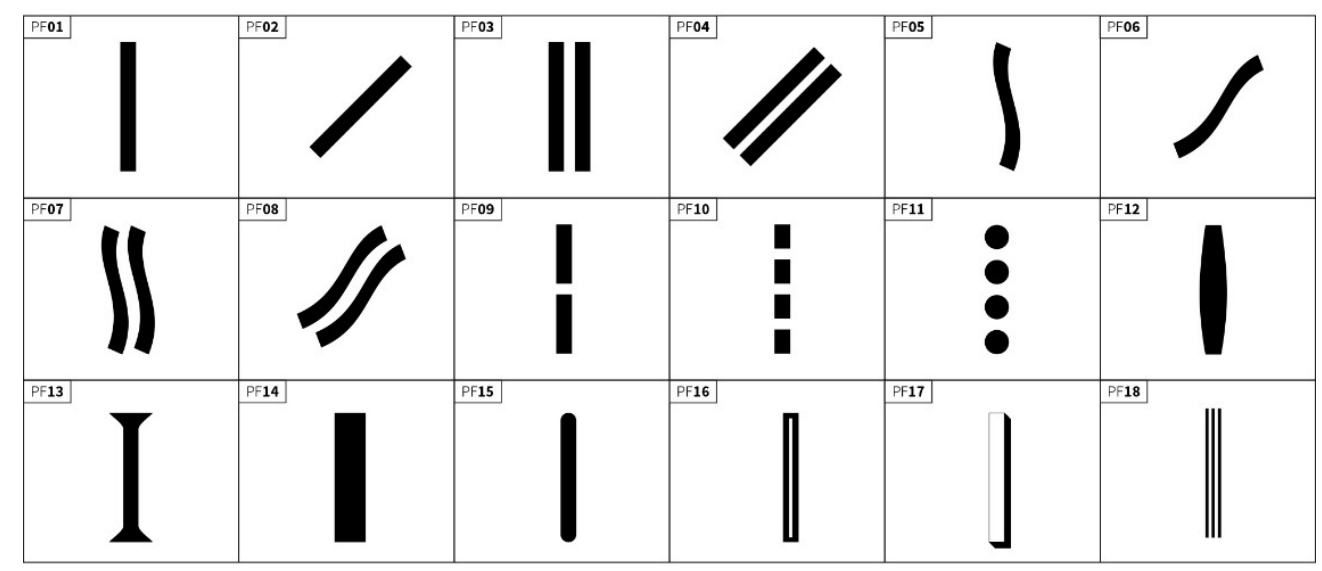

Figure 2: The formal attributes of letterforms of different complexity obtained with the qualitative assessment

The method of perimetric complexity was used to first measure the complexity of the constituent parts and then of the complete letterform. Due to diverse letterform properties, two metric systems which are different in its computational methods were chosen. Pelli's algorithm (Pelli, 2006) and the morphological components function (Watson, 2012) are both metrics for determining the perimetric complexity of binary digital images and are integrated into the computing system of the Wolfram Mathematica software. First, it was necessary to define an input binary image for both methods. In the case of Pelli's algorithm, the binary image is defined with values 0 and 1 where the value 0 represents foreground colour and value 1 background colour. The complexity of the perimeter is then defined by an average complexity value of the test samples. The morphological components function defines a binary image based on several separate regions which are label through integers. 


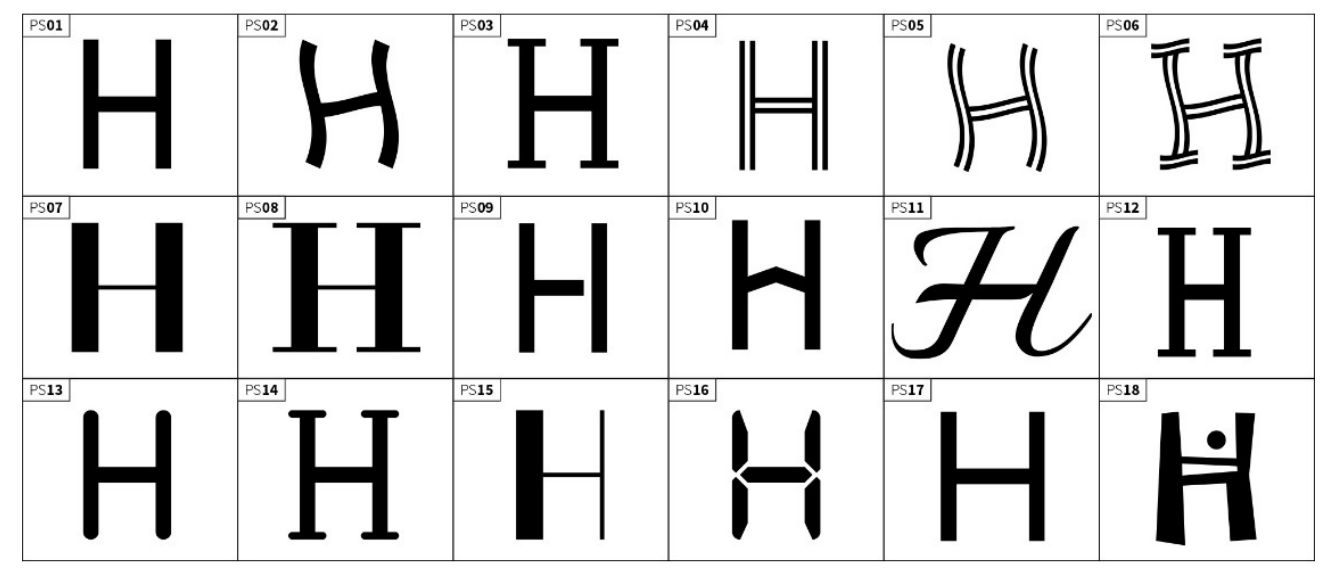

Figure 3: The formal attributes of letterforms of different complexity applied to the letterform $\mathrm{H}$

\section{RESULTS AND DISCUSSION}

The letterform characterization was performed using two metric systems for evaluation of the perimetric complexity. The analysis of the obtained results confirmed that particular formal attributes, singular or combined, are more complex than others, that is a gradient of letterform complexity was revealed. The first analysis focused on evaluation of the perimetric complexity of the formal attributes. The results of the perimetric complexity evaluation using Pelli's algorithm show that formal attributes which are vertical and slash can be more or less complex i.e. no evident gradation of the form's complexity is presented (Figure 4). When a single vertical line is expanded horizontally, has rounded terminals, is slashed/dashed or wavy the complexity of the form is low and the difference in the complexity value between the forms is insignificant. When a single vertical line is upgraded with serifs and double lines (vertical or slash) the perimetric complexity is increased. Also, the complexity value between each sample has a slight increase. However, making a line within a line, adding shadow or scribbles increases the complexity of the form significantly. Additionally, it can be observed that the increase of the complexity is steady up to the OF07 sample when it abruptly expands.

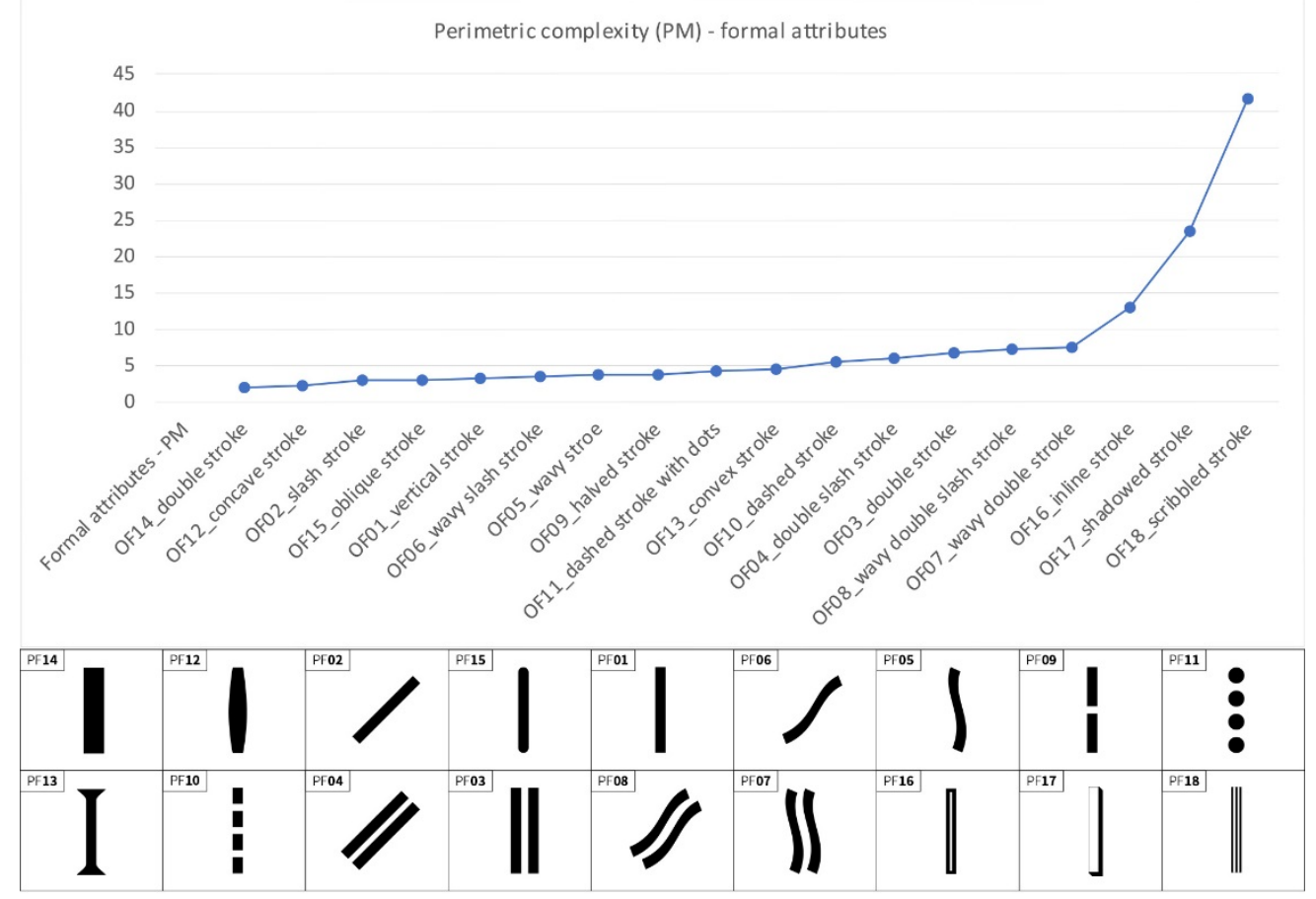

Figure 4: Graphic representation of the results of the perimetric complexity evaluation of the formal attributes using Pelli's algorithm 
The results of the perimetric complexity evaluation using the morphological components function showed slightly different results. It was observed that singular vertical stoke is less complex, that is particular variations of the vertical stroke (e.g. horizontal expansion, oblique terminals, dashed stroke) increase the form's complexity gradually. Additionally, the results reveal that the complexity is increased when the stoke shifts angularly, is doubled or wavy (Figure 5).

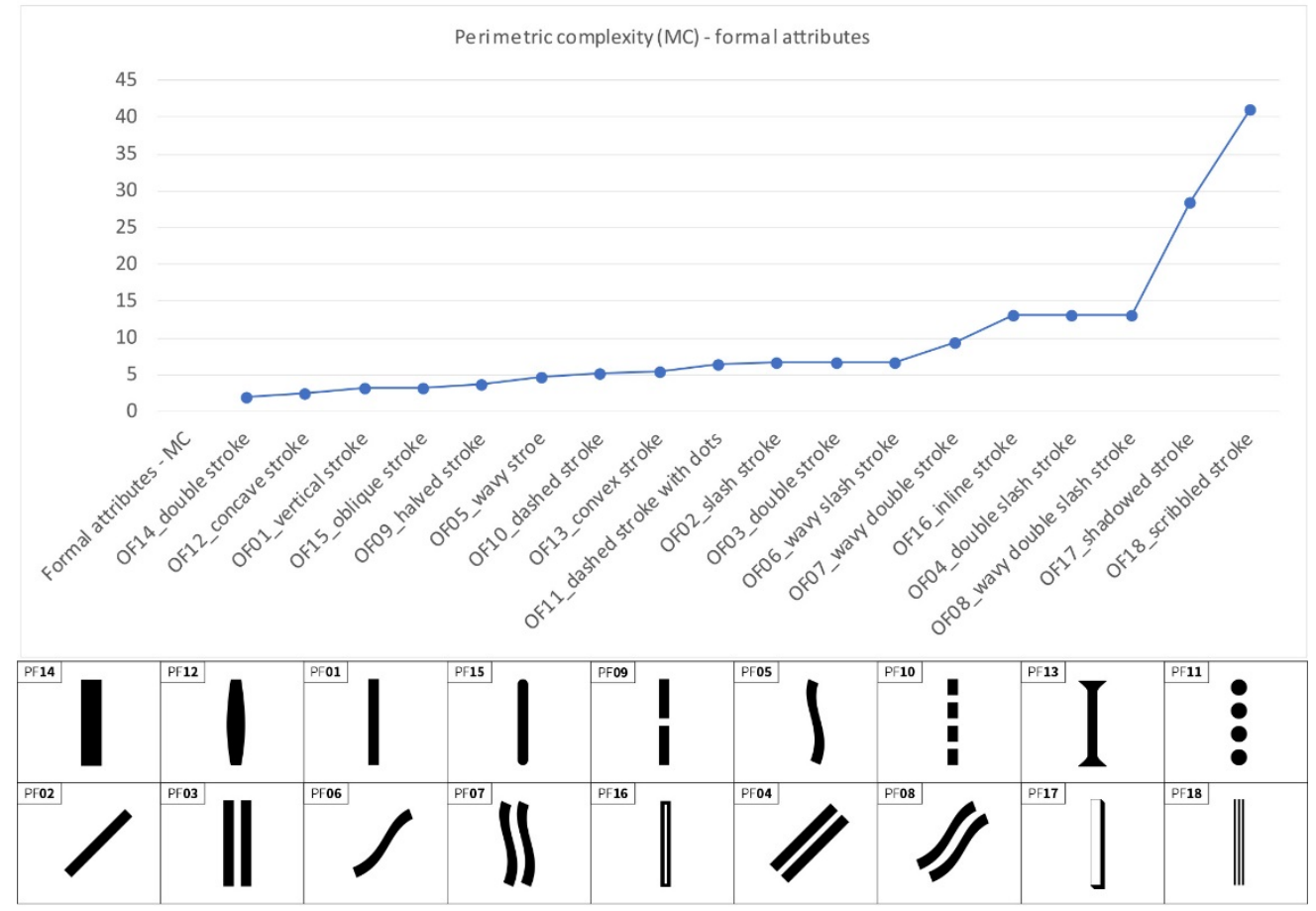

Figure 5: Graphic representation of the results of the perimetric complexity evaluation of the formal attributes using the morphological component function

In summary, it was observed that the morphological components function provides more conclusive results regarding the increase of the letterform perimetric complexity than Pelli's algorithm. Based on these results, it can be inferred that particular distinctive features i.e. formal attributes of letterforms are less or more complex. Also, the combination of particular attributes makes the form more complex where certain combinations expand the form's complexity drastically.

The second analysis focused on the evaluation of the perimetric complexity of the letterform $\mathrm{H}$ samples which were designed using the formal attributes from the first analysis. Figure 6 shows the results of the perimetric complexity using Pelli's algorithm. It can be observed that the complexity of the form is increased by combining the attributes. The attributes such as the letter contrast, proportions, oblique terminals, and irregular form have the less complex form while the letterform with serifs is more complex. A slight increase of the perimetric complexity is noticeable up to the OSO8 sample, whereas a significant increase is noticeable from this sample onwards. With the obtained results using this method it is difficult to form a conclusive grouping of the letterform complexity.

Figure 7 shows the results of the perimetric complexity using the morphological components function. Similarly, to the analysis of the singular formal attributes, this method provides much better results in regards to a gradual representation of the letterform complexity. It can be observed that letterforms constructed with dominantly vertical strokes tend to be less complex regardless of the letterform's contrast, proportions or the obliqueness of the terminals. On the other hand, serifs, double strokes or references to tools (script letterforms) increase perimetric complexity. A slight increase of the perimetric complexity is noticeable up to the OS16 sample after which the complexity increases more rapidly. Nevertheless, this method provides the gradation of the perimetric complexity that is increasing more steadily then in the case of the results obtained with Pelli's algorithm. 


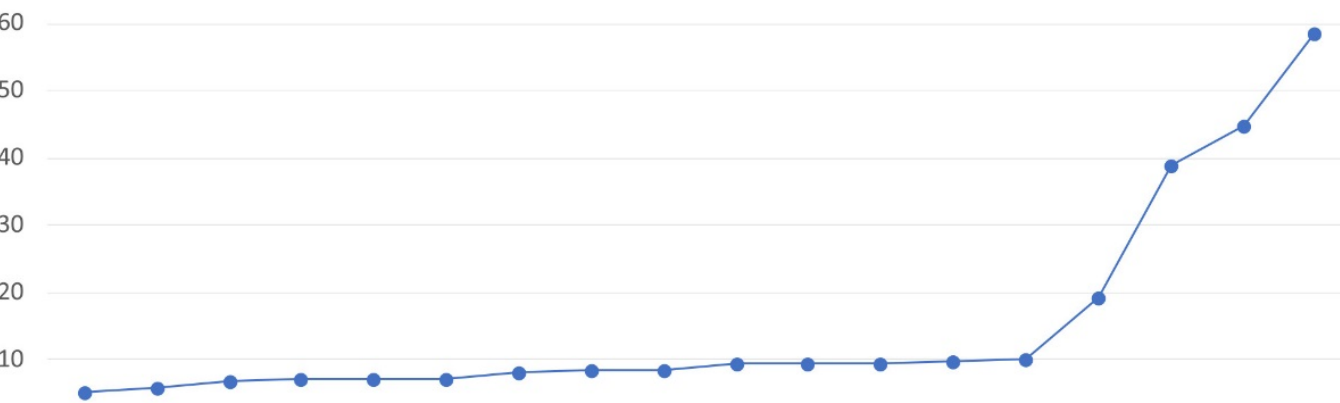

0

OSO9 OSO7 OS13 OSO1 OS10 OS17 OSO2 OS18 OS15 OS14 OS12 OS16 OSO3 OSO 8 OS11 OSO 4 OSO5 OSO

\section{НHНHHHHНHНHHНHҰHHH}

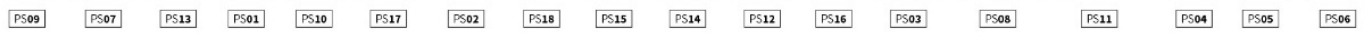

Figure 6: Graphic representation of the perimetric complexity evaluation for the letterform H using Pelli's algorithm

Perimetric complexity (MC) - letterform

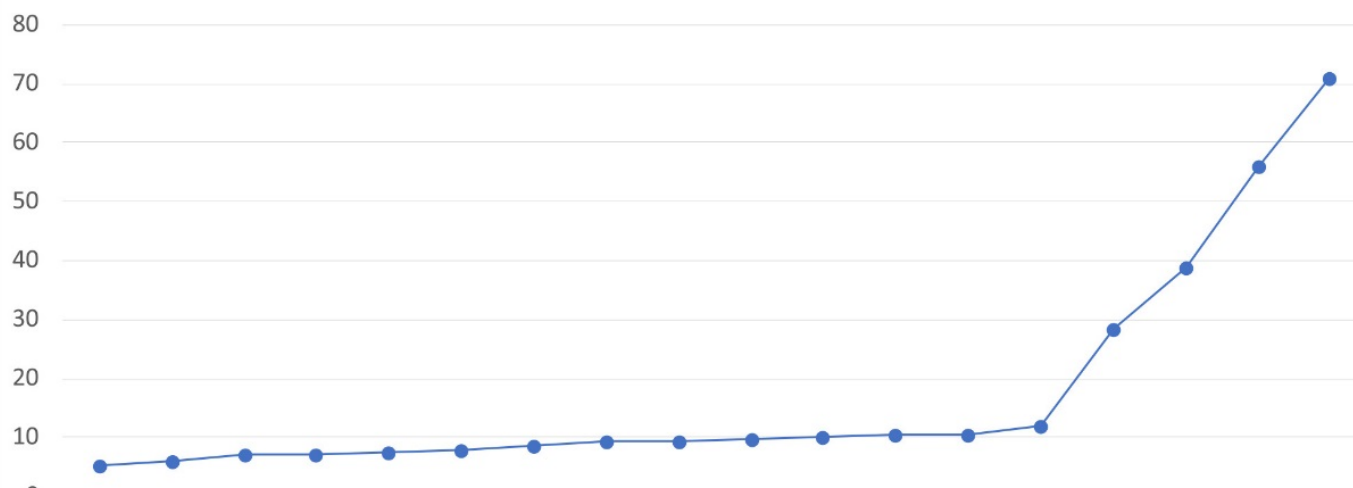

OS09 OS07 OSO1 OS13 OS17 OS10 OS15 OS18 OS12 OS03 OS14 OS08 OSO2 OS16 OS11 OSO 4 OS05 OSO6

\section{HHHHHHHHHHHHHFHHH

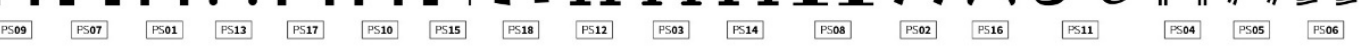

Figure 7: Graphic representation of the perimetric complexity evaluation for the letterform $\mathrm{H}$ using the morphological component function

\section{CONCLUSION}

The aim of this study was to propose a systematic method for manipulation of the typographic stimuli in research studies. The characterization of letterforms using the method of perimetric complexity has been proven functional and practical by the results of this study. Particularly, it was presented how the measurement of the letterform complexity, based on the quantitative analysis of the typeface formal attributes i.e. variations of the basic perceptual features successfully characterizes letterforms. Using the method of perimetric complexity, the results of the qualitative analysis were confirmed which revealed that combining the formal attributes in a single letterform provides a more complex structure. Additionally, the analysis in this study shows that one method of evaluating the perimetric complexity provides more conclusive results. The results of the letterform characterization using the morphological components function provide slightly more conclusive results where it was possible to observe that particular formal 
attributes combinations such as irregular forms, slashed or double strokes increase the letterform's complexity. These findings are relevant for future research in the field of the effects of typeface legibility and embellished typefaces where the methodology presented in this study will help obtain more consistent results across research studies.

\section{ACKNOWLEDGMENTS}

This work was supported by the Serbian Ministry of Science and Technological Development, Grant No.: 35027 "The development of software model for improvement of knowledge and production in graphic arts industry."

\section{REFERENCES}

[1] Beier, S., Larson, K.: "How does typeface familiarity affect reading performance and reader preference?", Information Design Journal, 20 (1), 16-31, 2013. doi: 10.1075/idj.20.1.02bei

[2] Beier, S., Sand, K., Starrfelt, R.: "Legibility implictations of embellished display typefaces", Visible Language, 51 (1), 112-133, 2017.

[3] Brumberger, E.R.: "The Rhetoric of Typography: The Persona of Typeface and Text", Technical Communication, 50 (2), 206-223, 2003.

[4] Childers, T.L., Jass, J.: "All Dressed Up With Something to Say: Effects of Typeface Semantic Associations on Brand Perceptions and Consumer Memory", Journal of Consumer Psychology, 12 (2), 93-106, 2002. doi: 10.1207/S15327663JCP1202_03

[5] Dixon, C.: "Describing typeforms: a designer's response", InfoDesign: Brazilian Journal of Information Design, 5 (2), 21-35, 2008.

[6] Fiset, D., Blais, C., Éthier-Majcher, C., Arguin, M., Bub, D., Gosselin, F.: “Features for uppercase and lowercase letter identification", Psychological Science, 19 (11), 1161-1168, 2008. doi: 10.1111/j.1467-9280.2008.02218.x

[7] Frutiger, A.: "Signs and symbols: their design and meaning", (Ebury Press, London, 1998).

[8] Henderson, P.W., Giese, J.L., Cote, J.A.: "Impression management using typeface design", Journal of Marketing, 68 (4), 60-72, 2004. doi: 10.1509/jmkg.68.4.60.42736

[9] Highsmith, C.: "Inside Paragraphs: Typographic Fundamentals", (The Font Bureau, Inc., Boston, 2012.)

[10] Lupton, E.: "Thinking with type", (New York, Princeton Architectural Press, 2004).

[11] Nedeljković, U.: "Univerzalno pismo modernistička utopija ili savremena komunikacijska potreba" PhD Thesis, University of Novi Sad, 2016.

[12] Nedeljković, S., Nedeljković, U.: "Pismo i tipografija", (FTN Izdavaštvo, Novi Sad, 2012).

[13] Nedeljković, U., Novaković, D., Pinćjer, I.: "Detecting universal structure and effects of typefaces", Technical Gazette, 24 (2), 557-564, 2017. doi: 10.17559/TV-20150831131738

[14] Pelli, D.G., Burns, C.W., Farell, B., Moore-Page, D.C.: "Feature detection and letter identification", Vision Research, 46 (28), 4646-4674, 2006. doi: 10.1016/j.visres.2006.04.023

[15] Rayner, K.: "Eye movements and attention in reading, scene perception, and visual search", The Quarterly Journal of Experimental Psychology, 62 (8), 1457-1506, 2009. doi: 10.1080/17470210902816461

[16] Rowe, C.L.: "The connotative dimensions of selected display typefaces", Information Design Journal, 3 (1), 30-37, 1982. doi: 10.1075/idj.3.1.03row

[17] Watson, A.B.: "Perimetric Complexity of Binary Digital Images", The Mathematica Journal, 14, 1-40, 2012. doi: $10.3888 /$ tmj.14-5

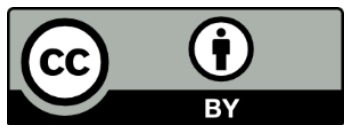

(C) 2018 Authors. Published by the University of Novi Sad, Faculty of Technical Sciences, Department of Graphic Engineering and Design. This article is an open access article distributed under the terms and conditions of the Creative Commons Attribution license 3.0 Serbia (http://creativecommons.org/licenses/by/3.0/rs/). 